\title{
Recent Advancements in Semiconductor-based Optical Signal Processing
}

\author{
Nielsen, M L; Mørk, Jesper
}

Published in:

European Conference on Optical Communications, 2006. ECOC 2006.

Link to article, DOI:

10.1109/ECOC.2006.4801175

Publication date:

2006

Document Version

Publisher's PDF, also known as Version of record

Link back to DTU Orbit

Citation (APA):

Nielsen, M. L., \& Mørk, J. (2006). Recent Advancements in Semiconductor-based Optical Signal Processing. In European Conference on Optical Communications, 2006. ECOC 2006. IEEE.

https://doi.org/10.1109/ECOC.2006.4801175

\section{General rights}

Copyright and moral rights for the publications made accessible in the public portal are retained by the authors and/or other copyright owners and it is a condition of accessing publications that users recognise and abide by the legal requirements associated with these rights.

- Users may download and print one copy of any publication from the public portal for the purpose of private study or research.

- You may not further distribute the material or use it for any profit-making activity or commercial gain

- You may freely distribute the URL identifying the publication in the public portal

If you believe that this document breaches copyright please contact us providing details, and we will remove access to the work immediately and investigate your claim 


\title{
Recent Advancements in Semiconductor-based Optical Signal Processing
}

M. L. Nielsen*, J. Mørk

COM•DTU, Technical University of Denmark, Oersteds Plads 345v, DK-2800 Kgs. Lyngby, Denmark

*Now with System Devices Research Laboratories, NEC Corporation, Japan (madsln@ap.jp.nec.com)

\begin{abstract}
Significant advancements in technology and basic understanding of device physics are bringing optical signal processing closer to a commercial breakthrough. In this paper we describe the main challenges in highspeed SOA-based switching.
\end{abstract}

\section{Introduction}

All-optical signal processing based on resonant nonlinearities in semiconductors has been heavily investigated for more than a decade. As a research topic it is still very active and the last couple of years have witnessed a number of significant advancements, e.g. the demonstration of wavelength conversion at $320 \mathrm{~Gb} / \mathrm{s}$ using a semiconductor optical amplifier (SOA) combined with a filter [1] and a monolithically integrated, potentially fast all-optical flip-flop [2]. These results illustrate two important points, namely 1) that the technology can certainly meet the bandwidth requirements for "simple", pipelining-like applications such as wavelength conversion, and 2) the path to micro-photonic, verylarge scale integration has been shown, with a flipflop footprint allowing for in excess of 100,000 components per square centimetre [3].

One of the main challenges in bringing optical signal processing to a commercial level is realizing simple, yet fast, schemes that can be integrated in large numbers (either monolithically or hybridly on a motherboard of a different substrate), while keeping the power consumption low. The latter is crucial, since signal processing will be carried out on a perchannel basis, which means that e.g. wavelength division multiplexing (WDM) routers will require a multitude of optical signal processing circuits. Since the electrical and optical power consumption increases with the bitrate it is imperative that the building blocks - the optical switches - use as little power as possible, since this will improve the scalability of the circuits.

Switches based on cross-phase modulation in SOAs have the best potential in terms of power consumption, since they provide gain for the switching signal [4]. In addition, they can be operated in a so-called differential mode [5], enabling operation at bitrates far beyond the inverse carrier lifetime. However, even in this case the impact of the finite carrier lifetime cannot be neglected, as it leads to a so-called nonlinear patterning (NLP) effect, which manifests as a fluctuation of the pulse energy of the switched bits [6]. Recent results show that the ultrafast nonlinearities play an important role in limiting the
NLP $[7,8]$.

In this paper we will review the recent advancements in SOA-based optical signal processing schemes and their integration, with an emphasis on approaches to increase the modulation bandwidth and reducing detrimental effects such as patterning effects and noise accumulation. We show examples from our own research on all-optical switches based on optical equalization of the slow SOA response.

\section{Patterning effects and noise}

The modulation of the carrier density in the active region of an SOA, and thus the patterning effect, is described by the carrier rate equation, which in its simplest form, in terms of the gain $g$, reads [6]

$$
\begin{aligned}
& \frac{d g}{d t}=-\frac{g(t)-g_{0}}{\tau_{e}}-k g(t) P_{c}(t) \\
& =-\frac{g(t)-g_{0}}{\tau_{e}}-k g_{0} P_{c}(t)-k \Delta g(t) P_{c}(t)
\end{aligned}
$$

where $k$ is a constant and $g_{0}, \tau_{e}, P_{c}(t)$, and $\Delta g(t)$ are the small-signal material gain, effective carrier lifetime, control signal, and gain change due to saturation, respectively.
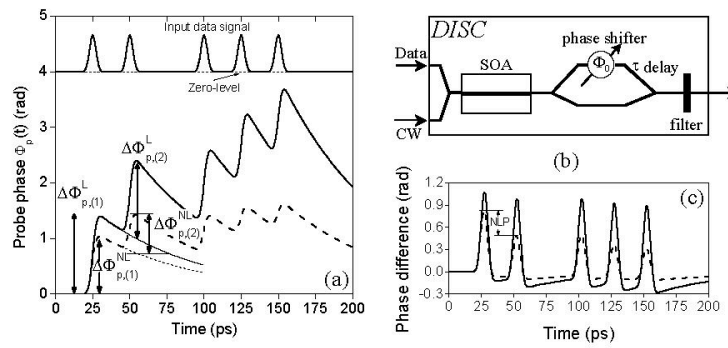

Fig. 1. (a) Calculated probe phase response to $40 \mathrm{~Gb} / \mathrm{s}$ contro/ signal, excluding nonlinear term in eq. (1) (solid), and including both terms (dashed). (b) DISC layout. (c) Phase difference between AMZI arms with (dashed) and without (solid) nonlinear term of eq. (1).

The two last terms of eq. (1) act as the driving terms for the dynamics of the gain. However, there is a major difference between the two, in that the first term, $k g_{0} P_{c}(t)$, is linear in the modulation, whereas the second term, $k \Delta g(t) P_{c}(t)$ is nonlinear. Ignoring the 
nonlinear term due to saturation, reduces eq. (1) to a linear equation, which can be analyzed using standard small-signal analysis of the carrier density modulation (CDM) [6]. A small-signal analysis accurately describes patterning effects attributable to the finite $\tau_{e}$, in cases where saturation does not influence the patterning, such as cross-gain modulation (XGM). This effect will be referred to as the linear patterming effect, which manifests as intersymbol interference. The problem of linear patterning can be reduced by launching a so-called holding beam into the SOA, which reduces the effective carrier lifetime $[9,10]$. Simply increasing the $\mathrm{CW}$ probe input power has a similar impact. However, another possibility is to operate SOA-based interferometric switches in the differential mode (DM) $[5,11]$, which effectively cancels the slow recovery of the carrier density induced gain and phase changes. This is illustrated in Fig. 1, where (a) shows the phase shift imposed on a continuous wave ( $\mathrm{CW}$ ) probe by a 40 $\mathrm{Gb} / \mathrm{s}$ control signal (shown in top inset) through crossphase modulation (XPM), in the case where the nonlinear term of eq. (1) is ignored (solid line), and the more realistic case where both terms are included (dashed line). Notice that when saturation is neglected, all control pulses provide the same phase shift, whereas this is not the case when saturation is taken into account. In the DM-switch referred to as the delayed-interference signal converter (DISC), Fig 1 (b), the phase modulated probe is passed through an asymmetric $M Z I$ (AMZI) filter, which converts the phase modulation into amplitude modulation. The transmittance of the filter is determined by the phase difference between the two arms, shown in Fig. 1 (c), excluding the nonlinear term (solid), and including both terms (dashed). It is observed that the linear patterning, induced by the linear driving term of eq (1), is completely compensated for after the AMZI, whereas the nonlinear term of eq. (1) introduces a clear modulation on the phase difference. The latter is the nonlinear patterning (NLP) effect, which is the only patterning effect remaining after elimination of linear patterning using the DM technique. NLP manifests itself as a fluctuation of the switched pulse power.

In [6] we presented a general approach to analyze the small-signal frequency response (SSFR) of an SOA followed by an optical filter, which optically equalizes the low-pass carrier density response (CDR) of the SOA. As a special case the DISC, i.e. an SOA+AMZI filter, has been analyzed, and the corresponding calculated SSFR is shown in the dashed curves of Fig. 2 for different values of the AMZI phase bias $\Phi_{0}$. The AMZI is observed to compensate for the lowpass CDR up to a bandwidth determined by the differential delay time, $\tau$, in the AMZI [6].

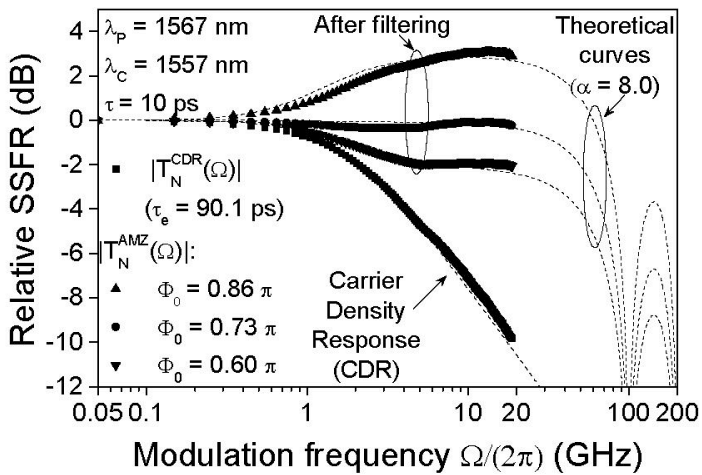

Fig. 2. Normalized carrier density response (CDR) and small-signal frequency response (SSFR) for DISC for different $\Phi_{0}$ values. Markers are measurements and dashed curves are theoretical, corresponding to $\alpha=8.0$.

Experimental data, also shown in Fig. 2, are measured for exactly the same values of phase bias $\Phi_{0}$, and are observed to match the theoretical results very well in the frequency range up to $20 \mathrm{GHz}$ that could be measured [12]. Notice the comparison with the low-pass CDR. Fig. 2 shows the SSFR normalized to the response at $\Omega=0$. To obtain a flat response up to ultra-high frequencies, the attenuated high frequency contents of the probe signal at the SOA output is enhanced by the AMZI, at the expense of the low-frequency components, which contain the most power. This leads to an inevitable trade-off between modulation bandwidth and optical signal-tonoise ratio (OSNR) $[6,13]$

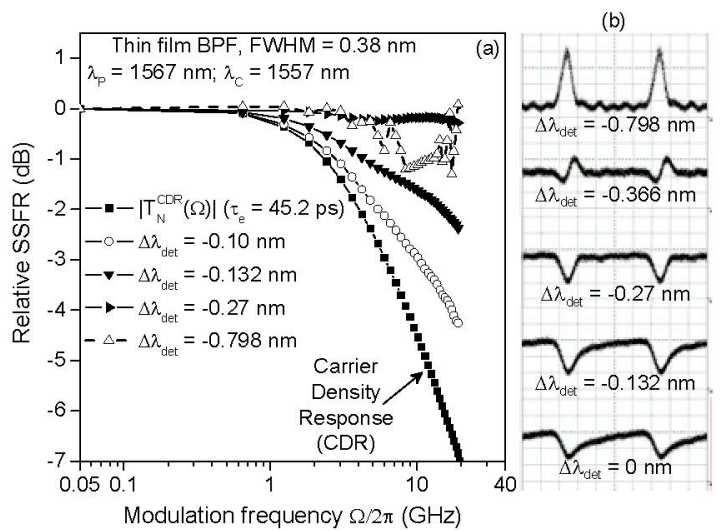

Fig. 3. (a) Normalized CDR and SSFR for switch consisting of SOA and BPF for different BPF detunings. (b) $10 \mathrm{GHz}$ large-signal response using same parameters as in (a).

The theory presented in [6] is completely general and can be applied to any optical filter. Fig. 3 (a) shows small-signal measurements for a $0.38 \mathrm{~nm}$ wide (FWHM) band-pass filter (BPF) when changing the detuning, $\Delta \lambda_{\text {det, }}$ towards the blue side. The normalized response becomes increasingly flat as $\left|\Delta \lambda_{\text {det }}\right|$ is increased, and is completely flat (up to 20 $\mathrm{GHz}$, at least) for a detuning of $-0.27 \mathrm{~nm}$. Theory predicts that the response becomes flat for a filter slope of $37.7 \mathrm{~dB} / \mathrm{nm}$, which is exactly the slope 
measured for a filter detuning of $-0.27 \mathrm{~nm}$ [7]. Fig. 3 (b) shows large-signal equivalents of the SSFR traces in Fig. 3 (b), in terms of switched output pulse traces, obtained by replacing the small-signal modulation by a $10 \mathrm{GHz}$ train of $2 \mathrm{ps}$ wide pulses. The impact of blue-shifting the BPF in the large-signal regime is observed by following the waveforms from the bottom and up: the recovery time is clearly reduced, and for $\Delta \lambda_{\text {det }}=-0.27 \mathrm{~nm}$ the inverted pulse is completely symmetric, indicating that the recovery time is as short as the fall-time. Increasing the blue-shift to $\Delta \lambda_{\text {det }}$ $=-0.366 \mathrm{~nm}$ and beyond, the polarity of the switched signal starts re-inverting, because the power in the carrier-peak, which determines the power in the marklevel of the inverted signal, is increasingly suppressed compared to the power in the blue sideband. This effect is also shown and explained in [13]. For $\Delta \lambda_{\text {det }}=$ $-0.798 \mathrm{~nm}$ the carrier peak is suppressed by roughly $25 \mathrm{~dB}$, which is sufficient to generate a switched signal with the same polarity as the control signal, and an extinction ratio of $8 \mathrm{~dB}$.

In [14], the complex filter transfer function required to restore the pulse shape of a switched $40 \mathrm{~Gb} / \mathrm{s}$ signal was calculated, and the filter successfully realized in MEMS technology. Another recently proposed switch configuration, which reduces the NLP imposed on the probe by the control signal has been dubbed the Turbo-Switch [15]. It involves passing the probe through an additional SOA after removing the control signal by a filter. Essentially, the second SOA works as high-pass filter, as described in e.g. [16,17], thereby speeding-up the total probe response. Using this scheme in a hybrid DISC configuration, wavelength conversion at $170 \mathrm{~Gb} / \mathrm{s}$ was demonstrated with a high output OSNR of $40 \mathrm{~dB} / 0.1$ $\mathrm{nm}$. The large OSNR is an advantage of this scheme over the, simpler, configuration of a single SOA and a detuned BPF, described above.

\section{Impact of ultra-fast dynamics}

Although it is possible to use the differential mode $[18,19]$ as well as the detuned filter approach $[20]$ to enhance the response of a switched NRZ signal, the lack of conveniently available NRZ signals above 50 $\mathrm{Gb} / \mathrm{s}$ has so far prevented a thorough analysis of NRZ switching. As a result, all ultra-high-speed (>50 Gb/s) demonstrations have been carried out with $\mathrm{RZ}$ control signals, typically generated from $10 \mathrm{GHz}$ trains of short pulses.

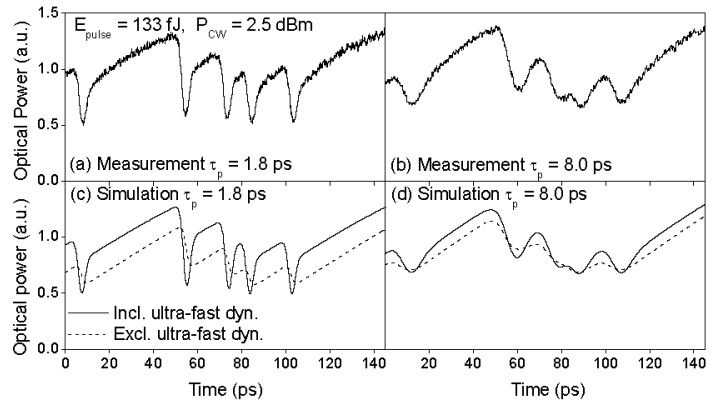

Fig. 4. Experimental $105 \mathrm{~Gb} / \mathrm{s}$ XGM traces for (a) $\tau_{p}=1.8 \mathrm{ps}$ and (b) $\tau_{p}=8.0 \mathrm{ps}$. Simulated traces incl. (solid) and excl. (dashed) ultra-fast dynamics for (c) $\tau_{p}=1.8$ and (d) $\tau_{p}=8.0$.

As analyzed in detail in [21,22], ultra-fast nonlinearities, such as carrier heating and spectral hole-burning, are excited when the control pulse width is below a certain critical width $\tau_{c r}$. Several independent references point to the importance of ultra-fast carrier dynamics in switching schemes based on an SOA followed by a filter $[7,8]$.

Fig. 4 (a) and (b) shows a comparison between experimentally obtained cross correlation traces of cross-gain modulated probe signals for data pulse widths of $\tau_{p}=1.8 \mathrm{ps}$ and $8 \mathrm{ps}$, respectively. In both cases the input is a periodic $105 \mathrm{~Gb} / \mathrm{s}$ " 1011010000 " data signal with an energy of $133 \mathrm{fJ} /$ pulse. In Fig. 4 (a) two distinct time scales are observed during gain recovery: an ultra-fast recovery immediately after the control pulses, followed by the much slower interband carrier relaxation. In Fig. $4(\mathrm{~b})$, where $\tau_{p} \approx \tau_{c r}$, the fast component is hardly visible. For comparison, traces simulated using the same input powers as in the experiment are shown in Fig. 4 (c) for $\tau_{p}=1.8 \mathrm{ps}$ and in Fig. 4 (d) for $\tau_{p}=8 \mathrm{ps}$. The XGM traces are shown with (solid) and without (dashed) ultra-fast effects to emphasize that the excellent agreement with the experimental traces relies on the inclusion of nonlinear gain suppression The scale is the same in all four plots.

The additional gain saturation caused by ultra-fast effects reduces the carrier density depletion by reducing the control pulse energy and thus the stimulated recombination rate [23]. The fact that the amount of carriers depleted per data pulse is diminished prompts a reduction of the carrier density dependent contribution to NLP for the DISC switch configuration (see Fig. 1). In addition, the ultra-fast dynamics can be shown to actually increase the switched output power and thereby the OSNR [7]. This, along with the AMZI filter's in-band noise filtering [24] ensures a satisfactory signal-to-noise ratio even at ultra-high bitrates. The current speed record for SOA-based switching is $320 \mathrm{~Gb} / \mathrm{s}$, which was obtained using a concatenation of detuned bandpass filters and an AMZI filter [1]. 


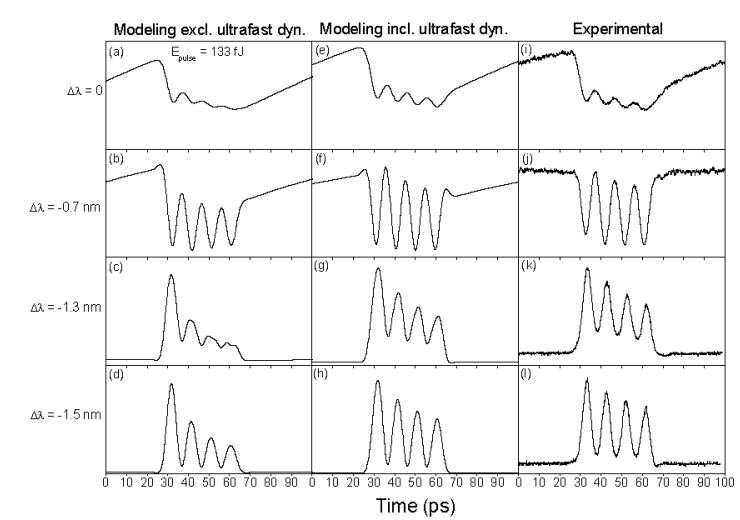

Fig. 5. Switched waveforms for SOA + detuned $0.9 \mathrm{~nm}$ wide $B P F$. Filter detuning is $0,-0.7 \mathrm{~nm},-1.3 \mathrm{~nm}$, and $-1.5 \mathrm{~nm}$ from top row to bottom row. First (second) column shows simulated waveforms excluding (including) ultra-fast effects, and third column shows corresponding experimental results.

This configuration was also found to be superior in [7].

Fig. 5 shows the impact of ultra-fast effects on a switch consisting of an SOA and a $0.9 \mathrm{~nm}$ wide (FWHM) BPF. The control signal is a $105 \mathrm{~Gb} / \mathrm{s}$ "1111000000" pattern consisting of 1.8 ps wide pulses. The left and middle columns show the modeled results excluding and including ultra-fast dynamics, respectively, and the right column shows the experimental result. The results are shown for four different filter detunings from the carrier of (from the top of Fig. 5) $0,-0.7 \mathrm{~nm},-1.3 \mathrm{~nm}$, and $-1.5 \mathrm{~nm}$. It is clear that ultra-fast dynamics also reduces NLP for this switch configuration. Moreover, the waveforms computed by including ultra-fast effects are in much better agreement with the experimental traces, which once again underlines the importance of including these effects

\section{Integration}

The switches described above are not all suitable for integration. However, the DM-MZI switch, including the differential delay, has recently been fabricated in an integrated version by several groups [4,25]. In [25] a $40 \mathrm{~Gb} / \mathrm{s}$ DM-MZI switch is monolithically integrated with the differential delay and a fast tunable laser, enabling fast packet forwarding. While monolithic integration provides obvious advantages, hybrid integration of nonlinear SOAs with a passive Silica interferometer reduces on-chip propagation losses by more than an order of magnitude [25], which reduces power consumption and improves the OSNR. In [26] a $40 \mathrm{~Gb} / \mathrm{s}$ hybrid DM-MZI is realized on a PLC MZI with a very low fiber-to-fiber excess loss $<2 \mathrm{~dB}$. Furthermore, a reduction of the control signal loss by $3 \mathrm{~dB}$ in each interferometer arm has been achieved by replacing the traditional $Y$-branch with $A M Z I$ filters/couplers [27].
Switches based on a single SOA and a sharp filter are difficult to integrate, since a sharp filter typically requires several filter stages, which makes it bulky and potentially lossy. However, the AMZI has been integrated with an SOA to realize an integrated version of the DISC switch, operating at $160 \mathrm{~Gb} / \mathrm{s}$ [28]. More complex filters have been used for FM $\rightarrow$ AM conversion in other contexts [29], so a sharp, asymmetric filter response, as the one exploited in [1], could be realized on-chip.

\section{Summary \& Discussion}

Recent advancements in all-optical, SOA-based signal processing have been reviewed, with an emphasis on simple, high-speed switches, which candidate as building blocks in future advanced optical processing circuits.

\section{References}

1. Y. Liu et al., OFC'06, PDP28 (2006)

2. M. T. Hill et al., Nature, 432 (2004)

3. M. Smit, Telektronikk 2 (2005)

4. A. Poustie, ECOC'05, WE3.5.1 (2005)

5. K. Tajima et al., JJAP 32, p. 1746 (1993)

6. M. L. Nielsen et al., JOSA B 21, p. 1606 (2004)

7. M. L. Nielsen et al., Opt. Express 14, p. 331 (2006)

8. Y. Liu et al., JLT 24 p. 230 (2006)

9. R. J. Manning et al., Opt. Lett. 19, p. 889 (1994)

10. S. Bischoff et al., JLT 22, p. 1303 (2004)

11. Y. Ueno, JOSA B 19, p. 2573 (2002)

12. M. L. Nielsen et al.,Opt.Express 14, p.1260 (2006)

13. M. L. Nielsen et al., Elect. Lett. 39, p. 1334 (2003)

14. J. Leuthold et al., JLT 22, p. 186 (2004)

15. R. J. Manning et al., OFC'06, OWS 8 (2006)

16. D. Marcenac, Intern. J. Optoelectr. 10, p.325 (1995)

17. M. L. Nielsen et al., JLT 18, p. 2151 (2000)

18. J. Leuthold et al.,Opt.Quan.Electr.33, p.939(2001)

19. K. Tajima et al., OFC'04, FD3 (2004)

20. M. L. Nielsen et al., OECC'05, 8F-1-2 (2005)

21. J. Moerk et al., JOSA B 19, p. 1803 (1996)

22. A. Mecozzi et al., JSTQE 3, p.1180 (1997)

23. J. Moerk et al., IEICE Trans.Electr. E87-C, p. 1126 (2004)

24. M. L. Nielsen et al., Opt. Express 13, p.5080 (2005)

25. V. Lal et al., ECOC'05 PDP 4.3.1 (2005)

26. G. Maxwell et al., ECTC'05 (2005)

27. G. Maxwell et al., ECOC'05 PDP 4.2.2 (2005)

28. J. Leuthold et al., Electr. Lett. 40, p. 554 (2004)

29. D. Mahgerefteh et al., Electr. Lett 41, p.43 (2005) 